\title{
SRAP Markers Associated with Water Use Efficiency and Some Agronomic Traits in Wheat under Different Irrigation Regimes
}

\author{
A.A. Said, A. Hamada ${ }^{*}$, M. Youssef ${ }^{* * \#}$, N.E. Mohamed and A.A. \\ Mustafa*** \\ Agronomy Department, Faculty of Agriculture, Sohag University, \\ Sohag; *Agronomy Department; ${ }^{* *}$ Genetics Department, Faculty \\ of Agriculture, Assiut University, Assiut and ${ }^{* * *}$ Soil \& Water \\ Department, Faculty of Agriculture, Sohag University, Sohag, \\ Egypt.
}

\begin{abstract}
TIFTY bread wheat lines were evaluated for drought tolerance and compared to six local cultivars under three water regimes (Wellwatered $=0.8$ Evapotranspiration $(\mathrm{ETp})$, Mild drought stress $=0.6 \mathrm{ETp}$ and severe drought stress $=0.4$ ETp.). Eight agronomic traits were evaluated, i.e. days to heading, number of spikes/plant, 100-kernel weight $(\mathrm{g})$, relative water content $(\%)$, chlorophyll concentration $\left(\mu \mathrm{g} \mathrm{cm}^{-2}\right)$, grain yield/plant $(\mathrm{g})$, harvest index and water use efficiency $\left(\mathrm{kg} / \mathrm{m}^{3}\right)$ under normal and stress conditions. Analysis of variance showed highly significant variations among the tested lines. As an average of all tested lines chlorophyll concentration was the most affected trait by drought followed by grain yield per plant and WUE, while harvest index showed the lowest reduction due to drought stress. Five lines $(1,5,11,41$ and 42) showed high performance in grain yield/plant and surpassed all local varieties under all conditions. The sequence related amplified polymorphism (SRAP) technique was used for the detection of markers associated with drought tolerance. SRAP was able to discriminate between the bulked-DNA of high and low performance lines in some evaluated traits under drought. Moreover, several unique and specific bands for high- and low-bulked lines were generated exposing the efficiency of SRAP in genotyping and diversity analysis. Evaluation of WUE showed its efficiency in differentiating among the tested lines and was in agreement with SRAP analysis which showed the maximum number of specific markers when the high- and low-WUE bulks were compared unlike the other traits. The generated bands could serve preliminarily as selectable markers for drought tolerance in wheat.
\end{abstract}

Keywords: Triticum aestivum, Water deficit, Molecular markers, Grain yield.

Wheat (Triticum aestivum L.) is one of the most important crops in the world especially in developing countries as it can be considered as the main source of carbohydrates. In Egypt, there is a big gap between consumption and production in wheat. To fill up this gap, wheat import reached about $57 \%$ of the total

\# Corresponding author Email: mkhirshy@ aun.edu.eg

Phone: (+2) (010) 98741898 
amount of wheat consumption (FAO, 2012). The total area of Egypt is about one million square kilometers, however out of this area only $3.7 \%$ can be used for living and farming due to limited water resources. In the last two decades Egypt population increased by about $84 \%$ (FAO, 2010), while the cultivated land and water resources still the same. To cover this increasing demand of wheat production, wheat could be cultivated under the limited water resources or even under drought condition

Stress conditions, such as drought, heat and salinity, are major problems that adversely affect wheat production. Drought is the main environmental abiotic stress, which has devastating effects on wheat productivity. Wheat production is adversely affected by drought in $50 \%$ and $70 \%$ of the area of the developed and the developing countries, respectively (Trethowan \& Pfeiffer, 2000). Hence, the introduction of varieties with improved tolerance to drought stress has been considered as one of the most important goals of crop improvement programs (Ludlow \& Muchow, 1990).

Drought tolerance is not a simple response, but is mostly conditioned by many components responses (Nazari \& Pakniyat, 2010). Most of agronomical characters are expressed differently in normal and stress conditions and are known to be affected by environmental factors. Therefore, selection based only on the phenotype would be difficult for such traits (Hittalmani et al., 2003).

Recently biotechnology provided powerful techniques to detect the molecular basis of plant adaptation to its environment and phenotype. The time has come to identify new strategies that combine advanced molecular technologies with conventional breeding and physiological techniques to achieve this goal. One of the most effective molecular markers is the sequence-related amplified polymorphism (SRAP) which is based on the amplification of open reading frames (ORFs) by targeting the exonic regions, intronic regions and regions with promoters (Li \& Quiros, 2001). SRAP markers are more reproducible, stable, simple, and more informative than other molecular markers.

In this study, we conducted two experiments to identify the effect of drought stress on yield and its components on some bread wheat genotypes. The first experiment was performed in the field under three levels of water stress and the second experiment was to differentiate between the highest and the lowest performance lines under molecular level using SRAP technique to detect markers associated with drought tolerance and then we can find an accurate tool for selection for drought tolerance at seedling stage, which will have a great impact on breeding programs for drought tolerance in wheat.

\section{Material and Methods}

Plant material

Fifty promising bread wheat lines were evaluated under different irrigation regimes. These lines were derived from two crosses as follow; 24 lines were derived from a cross between a high yielding local variety "Sids-4" with a

Egypt. J. Agron. 37, No. 2 (2015) 
drought tolerant variety "Tokwie" (South Africa) and 26 lines were derived from a cross between "Sids-4" and "Kasyon/glennson-81" (ICARDA). In addition, some local commercial varieties were used for agronomic evaluation comparison; those were Giza-164, Gemmiza-11, Sids-12, Shandawil-1, Masr-1 and Sahel-1.

\section{Phenotypic evaluation}

Two field experiments were carried out in the Experimental Farm of Faculty of Agriculture, Sohag University, Egypt, during 2012/2013 and 2013/2014 winter seasons. The experimental design was performed as randomized complete block combined over environments (irrigation treatments) and seasons with three replicates. The irrigation treatments were determined as different fractions of calculated potential evapotranspiration (ETp) in the experimental site, namely: Well-watered $=0.8 \mathrm{ETp}$, Mild drought stress $=0.6 \mathrm{ETp}$ and severe drought stress $=0.4 \mathrm{ETp}$. The experimental plot was consisted of two rows with two meters in length and $20 \mathrm{~cm}$ in between. Plants were individually spaced at $10 \mathrm{~cm}$ within each row. All cultural practices of growing wheat in the experimental location were followed as recommended. At harvesting, 10 guarded plants from each experimental plot were chosen at random and the following data were recorded: days to heading, No. of spikes/plant, 100-kernel weight, relative water content (RWC), chlorophyll concentration $\left(\mathrm{mg} \mathrm{cm}^{-2}\right)$ using SPAD chlorophyll meter and convert its reading using the formula $\left(y=0.118 x^{2}+0.919 x+7.925\right)$ described by Dash et al. (2007) as $\mathrm{y}=$ chlorophyll concentration $\left(\mathrm{mg} \mathrm{cm}^{-2}\right)$ and $x=$ SPAD reading, grain yield/plant, harvest index and water use efficiency (WUE).

\section{Climatic characteristics prevailing}

Monthly means of maximum and minimum temperature $\left(\mathrm{C}^{\circ}\right)$, relative humidity (RH) \%, wind speed (WS) $\mathrm{m} / \mathrm{sec}$, daily sunshine (DS) hours/day and evapotranspiration (ETo ) values were computed using ETo_Calculator_V3.2. FAO 2012 (Table 1).

\section{Soil characteristics of the experimental site}

Basic relevant physical and chemical characteristics of the experimental soil were determined according to Klute (1986) and Page (1982), respectively. Infiltration rate was determined by means of a double-ring infiltrometer (Model ASTM-D5093). The values are presented in Table 2.

\section{Irrigation requirement consumption and water supply}

The experimental plots were given volumes of water to raise the moisture of the top $45 \mathrm{~cm}$ layer to the field capacity. Water applied to the plots at each irrigation was equal to the difference between moisture at the field capacity and the soil moisture content at irrigation time of each irrigation (for each irrigation treatment) plus $10 \%$ of quantity to ensure a good uniform distribution of water through the plots (Table 3). 
TABLE 1. Averages of meteorological data and evapotranspiration reference (ETo) of the two growing seasons (2012/2013 and 2013/2014) .

\begin{tabular}{|l|c|c|c|c|c|c|c|}
\hline Measurement & Nov. & Dec. & Jan. & Feb. & Mar. & Apr. & Mean. \\
\hline Max. Temp. $\left(\mathrm{C}^{\mathrm{o}}\right)$ & 34.25 & 27.27 & 24.95 & 27.27 & 30.00 & 34.68 & 29.73 \\
\hline Min. Temp. $\left(\mathrm{C}^{\mathrm{o}}\right)$ & 6.38 & 2.79 & 5.00 & 5.43 & 7.00 & 8.82 & 5.90 \\
\hline RH (\%) & 58 & 63 & 65 & 64 & 51 & 37 & 56.33 \\
\hline WS (m/sec) & 1.6 & 1.4 & 1.3 & 1.6 & 1.9 & 1.9 & 1.62 \\
\hline DS (hours/day) & 9.3 & 9 & 8.9 & 9.8 & 9.9 & 10.3 & 9.53 \\
\hline ETo (mm/day) & 3.37 & 2.49 & 2.43 & 3.43 & 4.96 & 6.26 & 3.82 \\
\hline
\end{tabular}

TABLE 2. Physical and chemical properties of the experimental soil.

\begin{tabular}{|c|c|c|c|c|c|}
\hline \multicolumn{6}{|c|}{ Physical properties } \\
\hline Depth (cm) & $\begin{array}{c}\text { Bulk density } \\
\left(\mathrm{Mg} \mathrm{m}^{-3}\right)\end{array}$ & $\begin{array}{c}\text { Field } \\
\text { capacity } \\
(\%)\end{array}$ & $\begin{array}{c}\text { Permanent } \\
\text { wilting } \\
\text { point }(\%)\end{array}$ & $\begin{array}{c}\text { Available } \\
\text { water }(\%)\end{array}$ & Soil texture \\
\hline $0-15$ & 1.4 & 25 & 10 & 15 & Sandy clay loam \\
\hline $15-30$ & 1.4 & 24 & 9 & 15 & Sandy clay loam \\
\hline $30-45$ & 1.5 & 15 & 6 & 9 & Sandy loam \\
\hline \multicolumn{6}{|c|}{ Chemical properties } \\
\hline \multirow{2}{*}{\multicolumn{2}{|c|}{ Properties }} & \multicolumn{4}{|c|}{ Depth $(\mathrm{cm})$} \\
\hline & & \multicolumn{2}{|c|}{$00-30$} & \multicolumn{2}{|c|}{$30-60$} \\
\hline \multicolumn{2}{|l|}{ Soil pH } & \multicolumn{2}{|r|}{7.5} & \multicolumn{2}{|r|}{8.2} \\
\hline \multicolumn{2}{|c|}{$\mathrm{ECe}\left(\mathrm{dS} / \mathrm{m}\right.$ at $\left.25^{\circ} \mathrm{C}\right)$} & \multicolumn{2}{|r|}{2.1} & \multicolumn{2}{|r|}{2.5} \\
\hline \multicolumn{2}{|c|}{ Available nitrogen (ppm) } & \multicolumn{2}{|r|}{50} & \multicolumn{2}{|r|}{20} \\
\hline \multicolumn{2}{|c|}{ Available phosphorus (ppm) } & \multicolumn{2}{|r|}{20} & \multicolumn{2}{|r|}{22} \\
\hline \multicolumn{2}{|c|}{ Available potassium (ppm) } & \multicolumn{2}{|r|}{69} & \multicolumn{2}{|r|}{62} \\
\hline \multicolumn{2}{|c|}{$\mathrm{CaCO}_{3} \%$} & \multirow{2}{*}{\multicolumn{2}{|c|}{$\begin{array}{l}3.5 \\
1.9\end{array}$}} & \multicolumn{2}{|r|}{4.1} \\
\hline \multicolumn{2}{|c|}{ Organic matter $\%$} & & & & 1.4 \\
\hline
\end{tabular}

TABLE 3. Irrigation numbers, seasonal irrigation requirement and seasonal evapotranspiration for treatments in two seasons (2011/2012 and 2012/2013).

\begin{tabular}{|l|c|c|c|c|c|c|c|c|c|}
\hline Treatments & \multicolumn{2}{|c|}{ Number of irrigations } & \multicolumn{2}{|c|}{$\begin{array}{c}\text { Seasonal irrigation } \\
\text { requirement }\left(\mathbf{m}^{3} / \mathbf{f e d}\right)\end{array}$} & \multicolumn{2}{|c|}{$\begin{array}{c}\text { Seasonal evapo- } \\
\text { transpiration (mm) }\end{array}$} \\
\cline { 2 - 10 } & $\mathbf{2 0 1 2}$ & $\mathbf{2 0 1 3}$ & mean & $\mathbf{2 0 1 2}$ & $\mathbf{2 0 1 3}$ & mean & $\mathbf{2 0 1 2}$ & $\mathbf{2 0 1 3}$ & mean \\
\hline $\begin{array}{l}\text { Well- } \\
\text { watered }\end{array}$ & 12 & 12 & 12 & 2291 & 2302 & 2296.5 & 545.37 & 547.99 & 546.68 \\
\hline Mild stress & 9 & 9 & 9 & 2128 & 2125 & 2126.5 & 506.57 & 505.86 & 506.21 \\
\hline Severe stress & 6 & 6 & 6 & 1477 & 1487 & 1482.0 & 351.60 & 353.98 & 352.79 \\
\hline
\end{tabular}

Egypt. J. Agron. 37, No. 2 (2015) 


\section{Time of irrigation}

Daily evaporation data of pan ( $\mathrm{mm} /$ day) were obtained from a standard Class-A-Pan located in the experimental field, and recorded. Cumulative pan evaporation data for each irrigation treatment were calculated by: (multiplying daily evaporation by the studied evaporation pan coefficient) as following:

- Irrigation using 0.8 pan evaporation coefficient

- $\quad$ irrigation using 0.6 pan evaporation coefficient

- $\quad$ irrigation using 0.4 pan evaporation coefficient

Determination irrigation time was performed by setting the cumulative pan evaporation to be equal to the allowable available soil moisture depletion (50\%).

\section{Irrigation requirement computation and water supply}

Soil samples at three depths were collected directly before irrigation and after $48 \mathrm{hr}$ from irrigation. The quantity of water for each irrigation treatment was computed according to the following formula:

where :

$$
\mathrm{Q}=\mathrm{R} \times \mathrm{D} \times \text { Bd. } \times(\text { F.C. }- \text { S.M.I. }) / 100
$$

$\mathrm{Q}=$ the quantity of water in cubic meter, $\mathrm{R}=$ area that would be irrigated in square meter, $\mathrm{D}=$ the soil depth required to be irrigated in meter, $\mathrm{Bd}=$ bulk density of the soil $\left(\mathrm{gm} / \mathrm{cm}^{3}\right)$, F.C = field capacity of the experimental field in percent and S.M.I= the soil moisture percentage before irrigation.

Applied water was measured and delivered for each plot using water meter (Table 3).

\section{Evapotranspiration (ET), amount and rates}

The amount of evapotranspiration during irrigation cycle is assumed to be equal to the difference between both soil moisture contents after irrigation and before the next irrigation. The quantities of ET were calculated for $45 \mathrm{~cm}$ soil depth. For an area of $4200.8 \mathrm{~m}^{2}$ (one fed), evapotranspiration can be obtained by the following equation:

where:

$$
\mathrm{ET}=\theta_{2}-\theta_{1} / 100 \times \mathrm{Bd} \times \mathrm{D} / 100 \times 4200.8
$$

$\mathrm{ET}=$ evapotranspiration in $\mathrm{m}^{3}, \theta_{2}=$ soil moisture percent after irrigation, $\theta_{1}=$ soil moisture percent before next irrigation, $B d=$ bulk density in $\mathrm{gm} / \mathrm{cm}^{3}$ and $\mathrm{D}=$ soil depth in $\mathrm{cm}$.

Water use efficiency

WUE = grain yield $(\mathrm{kg} / \mathrm{fed}) /$ Seasonal ET $\left(\mathrm{m}^{3} / \mathrm{fed}\right),($ Vites, 1965)

Crop coefficient

$\mathrm{Kc}=\mathrm{ETc} / \mathrm{ETo} \quad$ (Allen et al., 1998) 
Statistical analysis

Analysis of variance (ANOVA), appropriate for the specified experimental design, was performed with MSTAT-C software to evaluate the genetic difference among the wheat genotypes. Statistical significance was assumed at 5 and $1 \%$ levels of probability. Differences among means were tested by least significant difference (LSD) test at $5 \%$ probability level.

\section{Molecular analysis}

DNA extraction

Genomic DNA from the fifty lines was extracted at seedling stage using Dellaporta et al. (1983) method with some modifications (Youssef, 2012). DNA concentration and purity were measured using spectrophotometer according to Stulnig \& Amberger (1994) and Khirshyat 1.0 micro-program (Youssef, 2012). Ten DNA samples of the highest and lowest lines in four agronomic traits evaluated under drought conditions were bulked and used for molecular analysis.

\section{SRAP-PCR amplification}

Ten SRAP primer combinations were selected and used for the molecular analysis (Table 4). The method of Li \& Quiros (2001) was followed for the SRAP marker system. Each $20 \mu \mathrm{l}$ SRAP amplification reaction consisted of $2 \mu 1$ of $10 \times$ PCR buffer, $1.6 \mu \mathrm{l}$ of $50 \mathrm{mM} \mathrm{MgCl} 2,1.6 \mu \mathrm{l}$ of $10 \mu \mathrm{M}$ of each forward and reverse primer, $2.5 \mu \mathrm{l}$ of 2 mMdNTPs, $25 \mathrm{ng}$ template DNA and $0.25 \mu \mathrm{l}$ of $5 \mathrm{U}$ Taq-DNA polymerase. The PCR was carried out with the initial cycle at $94^{\circ} \mathrm{C}$ for $2 \mathrm{~min}, 5$ cycles of $94^{\circ} \mathrm{C}$ for $30 \mathrm{~s}, 35^{\circ} \mathrm{C}$ for $30 \mathrm{~s}$ and $72^{\circ} \mathrm{C}$ for $1 \mathrm{~min}$, another 35 cycles of $94^{\circ} \mathrm{C}$ for $30 \mathrm{~s}, 50^{\circ} \mathrm{C}$ for $30 \mathrm{~s}$ and $72^{\circ} \mathrm{C}$ for $1 \mathrm{~min}$, and the final extension at $72^{\circ} \mathrm{C}$ for $5 \mathrm{~min}$. SRAP-PCR products were separated on $8 \%$ polyacrylamid gel (PAGE) and visualized by ethidium bromide.

TABLE 4. SRAP primer combinations used in molecular analysis.

\begin{tabular}{|l|c|c|}
\hline \multicolumn{2}{|c|}{ Forward (5-3 $)$} & Reverse (5'-3) \\
\hline 1 & Me-1: TGAGTCCAAACCGGATA & Em-3: GACTGCGTACGAATTGAC \\
\hline 2 & Me-3: TGAGTCCAAACCGGAAT & Em-2: GACTGCGTACGAATTTGC \\
\hline 3 & Me-3: TGAGTCCAAACCGGAAT & Em-3:GACTGCGTACGAATTGAC \\
\hline 4 & Me-4: TGAGTCCAAACCGGACC & Em-2:GACTGCGTACGAATTTGC \\
\hline 5 & Me-4: TGAGTCCAAACCGGACC & Em-3:GACTGCGTACGAATTGAC \\
\hline 6 & Me-5: TGAGTCCAAACCGGAAG & Em-5:GACTGCGTACGAATTAAC \\
\hline 7 & Me-3: TGAGTCCAAACCGGAAT & Em-5:GACTGCGTACGAATTAAC \\
\hline 8 & Me-4: TGAGTCCAAACCGGACC & Em-5:GACTGCGTACGAATTAAC \\
\hline 9 & Me-5: TGAGTCCAAACCGGAAG & Em-3:GACTGCGTACGAATTGAC \\
\hline 10 & Me-5: TGAGTCCAAACCGGAAG & Em-4:GACTGCGTACGAATTTGA \\
\hline
\end{tabular}

Molecular data analysis

SRAP profiles were converted to binary data matrices by detecting the presence (1) or the absence (0) of the strong, reproducible and clearly distinguished bands. The number of unique and specific bands for each agronomic trait was registered. The percentage of polymorphism was calculated by dividing the total number of polymorphic bands on the total number of bands.

Egypt. J. Agron. 37, No. 2 (2015) 
Analysis of variance

\section{Results}

The combined analysis of variance for days to heading, number of spikes/plant, 100-kernel weight $(\mathrm{g})$, relative water content $(\%)$, chlorophyll concentration $\left(\mu \mathrm{g} \mathrm{cm} \mathrm{cm}^{-2}\right)$, grain yield/plant $(\mathrm{g})$, harvest index and water use efficiency $(\mathrm{kg} / \mathrm{m})$ revealed that the effect of highly significant affected by years, water stress treatments and genotypes.

TABLE 5. Mean squares of the combined analysis of variance for all studied traits.

\begin{tabular}{|c|c|c|c|c|c|c|c|c|c|}
\hline \multirow[b]{2}{*}{ S.o.V } & \multirow[b]{2}{*}{ D.F } & \multicolumn{8}{|c|}{ Mean Squares } \\
\hline & & Heading date & $\begin{array}{c}\text { Number of } \\
\text { spikes / plant }\end{array}$ & $\begin{array}{l}100 \text { kernel } \\
\text { weight }\end{array}$ & RWC & $\begin{array}{c}\text { Chlorophyll } \\
\text { content }\end{array}$ & $\begin{array}{c}\text { Grain yield / } \\
\text { plant }\end{array}$ & Harvest index & WUE \\
\hline $\operatorname{Year}(\mathbf{Y})$ & 1 & $765.35^{* *}$ & $19.86^{* *}$ & $5.99 * *$ & $697.22^{* *}$ & $91802.82 * *$ & $670.49 * *$ & $256.58^{* *}$ & $1.09 * *$ \\
\hline Drought (D) & 2 & $8824.12^{* *}$ & $412.11^{* *}$ & $303.13 * *$ & $16302.02 * *$ & $2957028.13^{* *}$ & $4819.56^{* * *}$ & $392.10^{* *}$ & $8.90^{* *}$ \\
\hline Y x D & 2 & 2.49 & 1.45 & $1.33^{* *}$ & 7.25 & $3758.59^{*}$ & 21.69 & $41.96^{*}$ & 0.007 \\
\hline Error a & 12 & 4.2 & 0.748 & 0.473 & 10.89 & 129.06 & 7.39 & 9.14 & 0.0033 \\
\hline Genotype (G) & 55 & 156.40 ** & $26.46^{* *}$ & $1.59 * *$ & $152.55^{* *}$ & $125698.92^{* * *}$ & $73.84 * *$ & $178.09 * *$ & $0.131^{* *}$ \\
\hline Y x G & 55 & $3.43^{* *}$ & $0.616^{* *}$ & $0.520 * *$ & 0.512 & $810.01^{* *}$ & $6.93 * *$ & $22.55^{* *}$ & $0.0069 * *$ \\
\hline D x G & 110 & $12.14^{* *}$ & $2.59^{* *}$ & $0.533^{* *}$ & $40.70^{* *}$ & $14344.37^{* *}$ & $18.35^{* *}$ & $33.71 * *$ & $0.029 * *$ \\
\hline$Y \times D \times G$ & 110 & $1.22 * *$ & $0.501^{* *}$ & $0.207 * *$ & 0.407 & $778.17 * *$ & $1.88 * *$ & $6.92 * *$ & 0.0016 \\
\hline Error b & 660 & 0.412 & 0.23 & 0.02 & 2.27 & 150.1 & 0.629 & 3.28 & 0.00097 \\
\hline
\end{tabular}

$* \& * *$ Significant at $5 \%$ and $1 \%$ levels of probability, respectively.

The results revealed that wheat genotypes varied from each other for all traits under the three water applications (Table 6).

TABLE 6. The range and mean values for all studied traits under well-watered, mild stress and severe stress conditions.

\begin{tabular}{|c|c|c|c|c|c|c|}
\hline \multirow{2}{*}{ Traits } & \multicolumn{2}{|c|}{ Well-watered } & \multicolumn{2}{|c|}{ Mild stress } & \multicolumn{2}{|c|}{ Sever stress } \\
\hline & Range & Mean \pm S.E & Range & Mean \pm S.E & Range & Mean \pm S.E \\
\hline Heading date & $75.83-91.50$ & $85.71 \pm 0.20$ & $72.83-84.50$ & $79.72 \pm 0.22$ & $70.50-79.00$ & $75.67 \pm 0.20$ \\
\hline No. of spikes/plant & $6.63-14.64$ & $9.69 \pm 0.20$ & $5.86-10.58$ & $8.13 \pm 0.22$ & $4.99-9.65$ & $7.51 \pm 0.20$ \\
\hline 100 kernel weight (g) & $4.78-6.16$ & $5.39 \pm 0.02$ & $3.82-5.81$ & $4.65 \pm 0.02$ & $2.64-4.31$ & $3.50 \pm 0.02$ \\
\hline RWC \% & $75.47-88.64$ & $84.76 \pm 0.07$ & $69.84-81.61$ & $76.82 \pm 0.08$ & $57.14-78.53$ & $70.88 \pm 0.07$ \\
\hline $\begin{array}{l}\text { Total chlorophyll content } \\
(\mu \mathrm{g} \mathrm{cm}-2)\end{array}$ & $32.20-68.02$ & $47.09 \pm 0.12$ & $24.17-55.24$ & $37.12 \pm 0.12$ & $19.29-47.74$ & $30.61 \pm 0.12$ \\
\hline Grain yield/plant (g) & $11.54-28.50$ & $18.51 \pm 0.02$ & $9.77-17.72$ & $12.98 \pm 0.02$ & $7.43-14.84$ & $11.10 \pm 0.02$ \\
\hline Harvest index & $28.15-46.35$ & $33.71 \pm 0.12$ & $26.22-40.64$ & $31.71 \pm 0.12$ & $24.07-41.05$ & $31.80 \pm 0.12$ \\
\hline WUE (kg/m) & $0.52-1.21$ & $0.82 \pm 0.12$ & $0.44-0.77$ & $0.59 \pm 0.12$ & $0.33-0.67$ & $0.50 \pm 0.12$ \\
\hline
\end{tabular}

Egypt. J. Agron. 37, No. 2 (2015) 


\section{Performance of wheat genotypes}

The average number of days to heading in the two years ranged from 70.50 days for line No. 47 in severe drought condition to 91.50 days for line No. 28 in well-watered condition. The average of overall tested genotypes for number of days to heading was reduced from 85.66 days in well-watered condition to 79.92 days in mild stress condition and down to 75.44 days in severe drought condition. The earliest lines were No. 18, 19, 29, 37, 45, 46, 47 and 49 as compared with the earliest local variety under all stress conditions (Table 7).

Severe stress reduced the number of spikes/plant by $22 \%$ as an average for all tested genotypes when compared with well-watered conditions (Table 7). Results showed that lines No. 26 and 41 surpassed the check (Sahel 1) and local commercial varieties in No. of spikes/plant under all conditions.

Drought stress at grain filling adversely affected 100-kernel weight (g). As an average for all tested genotypes, the reduction ranged from $14 \%$ to $35 \%$ due to mild and severe drought conditions, respectively. Only line No. 28 was significantly higher than the check (Sahel 1) and local commercial varieties under all conditions. While, lines No. 1, 4, 7, 8, 9, 11, 30, 35 and 39 were significantly surpassed the check (Sahel 1) and local commercial varieties under severe stress conditions.

Water stress cases a reduction on relative water content (RWC). In this regard, as overall average of all genotypes RWC was reduced from $84.90 \%$ in well-watered conditions to $76.88 \%$ in mild stress and the severe conditions recorded the lowest value $71.11 \%$ (Table 7). Lines No. 1, 41 and 42 were significantly exceeded the check (Sahel 1) and local commercial varieties under all conditions. While, nine lines, i.e., line No. 5, 10, 11, 16, 19, 22, 23, 34 and 43 surpassed the check (Sahel 1) and local commercial varieties in RWC under mild and severe stresses conditions.

Under well-watered condition the average chlorophyll concentration $\left(\mathrm{mg} \mathrm{cm}^{-2}\right.$ ) was 344.33 with a range from 616.43 to 160.02 for lines No. 11 and 6, respectively. Meanwhile, there was a reduction in chlorophyll concentration about $36 \%$ and $54 \%$ caused by mild and severe conditions, respectively (Table 8). Results showed that seven lines, i.e., No. 1, 2, 11, 37, 41, 42 and 43 surpassed the check (Sahel 1) and local commercial varieties in chlorophyll concentration over all conditions. 
TABLE 7. Mean performance of heading date, number of spikes/plant, 100-kernel weight and relative water content .

\begin{tabular}{|c|c|c|c|c|c|c|c|c|c|c|c|c|}
\hline \multirow[b]{2}{*}{ Genotypes } & \multicolumn{3}{|c|}{ Heading date } & \multicolumn{3}{|c|}{ Number of spikes / plant } & \multicolumn{3}{|c|}{ 100-kernel weight } & \multicolumn{3}{|c|}{ Relative water content } \\
\hline & $\begin{array}{c}\text { Well- } \\
\text { watered }\end{array}$ & $\begin{array}{c}\text { Mild } \\
\text { stress }\end{array}$ & $\begin{array}{c}\text { Sever } \\
\text { stress }\end{array}$ & $\begin{array}{c}\text { Well- } \\
\text { watered }\end{array}$ & $\begin{array}{c}\text { Mild } \\
\text { stress }\end{array}$ & $\begin{array}{l}\text { Sever } \\
\text { stress }\end{array}$ & $\begin{array}{c}\text { Well- } \\
\text { watered }\end{array}$ & $\begin{array}{c}\text { Mild } \\
\text { stress }\end{array}$ & $\begin{array}{l}\begin{array}{l}\text { Sever } \\
\text { stress }\end{array} \\
\end{array}$ & $\begin{array}{c}\text { Well- } \\
\text { watered }\end{array}$ & $\begin{array}{c}\text { Mild } \\
\text { stress }\end{array}$ & $\begin{array}{l}\begin{array}{l}\text { Sever } \\
\text { stress }\end{array} \\
\end{array}$ \\
\hline 1 & 89.00 & 83.50 & 79.00 & 10.81 & 8.57 & 7.71 & 5.73 & 5.01 & 4.11 & 88.22 & 79.81 & 75.56 \\
\hline 2 & 86.50 & 82.33 & 77.17 & 10.97 & 9.60 & 8.72 & 5.21 & 4.81 & 3.83 & 87.05 & 79.70 & 75.11 \\
\hline 3 & 86.17 & 80.50 & 75.67 & 10.35 & 7.85 & 7.26 & 5.30 & 4.17 & 3.46 & 84.70 & 73.34 & 69.59 \\
\hline 4 & 90.00 & 82.00 & 79.00 & 11.73 & 9.79 & 9.14 & 5.61 & 4.77 & 4.06 & 86.23 & 76.61 & 67.53 \\
\hline 5 & 89.83 & 81.83 & 77.67 & 9.54 & 7.69 & 7.47 & 5.25 & 4.65 & 3.47 & 86.27 & 78.99 & 75.67 \\
\hline 6 & 85.50 & 79.50 & 77.33 & 8.49 & 6.86 & 6.68 & 5.43 & 4.61 & 3.78 & 81.29 & 76.28 & 66.87 \\
\hline 7 & 88.83 & 84.17 & 77.17 & 9.83 & 7.86 & 7.58 & 5.25 & 4.84 & 3.99 & 87.85 & 76.60 & 68.19 \\
\hline 8 & 87.00 & 83.50 & 78.33 & 8.93 & 8.66 & 8.08 & 5.31 & 4.59 & 4.02 & 82.44 & 78.61 & 70.19 \\
\hline 9 & 86.00 & 80.00 & 73.67 & 9.07 & 7.60 & 6.80 & 5.71 & 4.98 & 4.00 & 87.46 & 72.76 & 59.34 \\
\hline 10 & 84.11 & 77.17 & 74.00 & 7.80 & 7.96 & 7.50 & 5.37 & 4.64 & 3.58 & 83.39 & 80.95 & 77.54 \\
\hline 11 & 90.17 & 83.00 & 78.00 & 10.29 & 8.56 & 8.03 & 5.57 & 5.02 & 4.15 & 87.48 & 80.07 & 76.32 \\
\hline 12 & 85.00 & 79.67 & 74.67 & 10.61 & 7.08 & 6.91 & & 4.99 & 3.82 & & 74.48 & 70.06 \\
\hline 13 & 83.17 & 77.33 & 73.00 & 9.24 & 8.67 & 8.19 & 5.30 & 4.41 & 2.67 & 83.09 & 72.15 & 67.73 \\
\hline 14 & 85.50 & 81.50 & 73.67 & 11.21 & 10.08 & 9.45 & 5.21 & 4.50 & 3.47 & .70 & 79.70 & 3.95 \\
\hline 15 & 85.67 & 82.17 & 78.50 & 11.38 & 9.83 & 9.08 & 5.34 & 75 & 3.50 & & 76.83 & 2.74 \\
\hline 16 & 90.83 & 83.83 & 77.00 & 7.97 & 6.33 & 6.05 & 5.20 & 87 & 3.7 & & 78.90 & 6.32 \\
\hline 17 & 87.50 & 81.83 & 78.33 & 11.63 & 9.48 & 8.48 & 5.28 & 4.61 & 3.77 & 39 & 74.22 & 68.47 \\
\hline 18 & 80.50 & 75.67 & 72.33 & 8.44 & 7.38 & 6.24 & 4.99 & 4.46 & 3.56 & .73 & 75.04 & 60.95 \\
\hline 19 & 79.83 & 74.50 & 73.00 & 9.30 & 8.49 & 7.88 & 5.59 & 5.17 & 2.77 & .29 & 80.34 & 76.59 \\
\hline 20 & 82.50 & 77.83 & 74.33 & 7.64 & 8.08 & 7.40 & 5.31 & 4.05 & 3.40 & 83.87 & 79.59 & 72.84 \\
\hline 21 & 83.83 & 78.33 & 73.67 & 8.19 & 6.57 & 5.74 & 5.55 & 4.94 & 2.64 & 82.60 & 71.22 & 57.14 \\
\hline 22 & 88.50 & 83.50 & 79.00 & 10.48 & 8.54 & 8.00 & 5.43 & 4.70 & 3.64 & 86.67 & 80.51 & 77.43 \\
\hline 23 & 88.50 & 81.17 & 76.67 & 10.25 & 7.44 & 7.26 & 5.89 & 5.01 & 2.68 & 85.63 & 78.81 & 76.72 \\
\hline 24 & 88.83 & 84.50 & 77.00 & 9.21 & 8.40 & 7.13 & 4.78 & 4.16 & 3.81 & 82.81 & 71.97 & 66.55 \\
\hline 25 & 85.50 & 78.67 & 75.00 & 10.78 & 9.74 & 7.52 & 5.04 & 4.00 & 3.13 & 81.88 & 76.83 & 72.08 \\
\hline 26 & 91.17 & 80.50 & 74.67 & & & 8.80 & & 4.95 & & & & 71.16 \\
\hline 27 & 87.33 & 78.50 & 74.00 & & & & & 83 & & & & 9.57 \\
\hline 28 & 91.50 & 80.67 & 76.67 & 12.30 & 9.09 & 7.28 & 12 & & & & & 6.73 \\
\hline 29 & 79.83 & 76.50 & 72.83 & 8.15 & 8.00 & 7.74 & 5.42 & 27 & 3. & & 78.26 & 74.98 \\
\hline 30 & 89.17 & 79.00 & 75.00 & 10.46 & 7.33 & 6.91 & 5.46 & 11 & 4.3 & 95 & 69.84 & 64.09 \\
\hline 31 & 88.17 & 81.50 & 77.00 & 10.52 & 8.23 & 8.22 & 5.44 & .73 & 3.07 & & 77. & 9.39 \\
\hline 32 & 91.17 & 81.50 & 77.33 & 7.81 & 7.02 & 7.00 & 5.34 & 96 & 3.7 & & 75. & 71.11 \\
\hline 33 & 88.67 & 84.00 & 78.67 & 9.22 & 9.22 & 7.83 & 5.0 & 43 & 3.3 & & & 71.41 \\
\hline 34 & 84.67 & 80.67 & 74.33 & 9.01 & 8.98 & 8.44 & 5.02 & 4.40 & 3.30 & 87.26 & 80.87 & 76.79 \\
\hline 35 & 85.17 & 79.50 & 74.67 & 10.74 & 8.72 & 7.83 & 5.63 & 5.23 & 4.33 & 83.30 & 73.81 & 67.06 \\
\hline 36 & 82.83 & 76.17 & 72.67 & 8.52 & 7.44 & 7.04 & 5.27 & 4.37 & 3.10 & 79.47 & 73.97 & 63.88 \\
\hline 37 & 79.83 & 75.33 & 73.00 & 10.34 & 8.71 & 8.49 & 5.49 & 4.69 & 3.68 & 86.61 & 79.80 & 74.54 \\
\hline 38 & 90.17 & 81.50 & 77.00 & 8.71 & 8.36 & 7.93 & 5.62 & 4.52 & 2.80 & 81.78 & 76.76 & 71.68 \\
\hline 39 & 84.50 & 78.33 & 73.67 & 9.98 & 7.19 & 6.89 & 5.33 & 4.82 & 4.16 & 86.57 & 73.88 & 64.13 \\
\hline 40 & 86.50 & 78.83 & 75.67 & 9.35 & 6.95 & 6.8 & 5.49 & 4.24 & 3.5 & 85.93 & 77.81 & 73.06 \\
\hline 41 & 90.00 & 83.50 & 77.67 & 14.64 & 10.58 & 9.6 & 5.81 & 36 & & 3.64 & & 6.75 \\
\hline 42 & 86.50 & 79.83 & 74.33 & 12.27 & 9.71 & 8.2 & 5.4 & 4.02 & 3. & 87.56 & 79.83 & 76.25 \\
\hline 43 & 86.50 & 80.33 & 75.00 & 7.60 & 6.61 & 6.9 & 5.7 & 4.40 & 3.28 & 86.35 & 81.61 & 78.53 \\
\hline 44 & 80.89 & 76.50 & 73.00 & 9.28 & 7.91 & 7.9 & 5.1 & 4.86 & 3.0 & & 75.45 & 71.95 \\
\hline 45 & 77.83 & 72.83 & 70.67 & 7.53 & 7.77 & 7.26 & 5.47 & 4.47 & 2.9 & 80.58 & 76.16 & 73.08 \\
\hline 46 & 78.83 & 73.67 & 71.00 & 7.78 & 6.74 & 6.02 & 5.37 & 4.51 & 3.48 & 85.91 & 80.02 & 66.60 \\
\hline 47 & 75.83 & 72.83 & 70.50 & 6.63 & 5.86 & 4.99 & 4.80 & 3.82 & 2.59 & 75.47 & 73.17 & 65.53 \\
\hline 48 & 87.17 & 82.50 & 77.17 & 8.28 & 6.80 & 6.68 & 5.03 & 4.23 & 3.27 & 84.42 & 77.25 & 70.84 \\
\hline 49 & 76.50 & 73.50 & 72.67 & 8.15 & 7.13 & 5.85 & 4.91 & 4.51 & 3.16 & 84.52 & 75.70 & 69.29 \\
\hline 50 & 85.83 & 79.83 & 74.67 & 9.66 & 7.95 & 7.43 & 6.01 & 5.13 & 2.88 & 84.45 & 79.08 & 74.22 \\
\hline Mean & 85.71 & 79.72 & 75.34 & 9.69 & 8.13 & 7.51 & 5.40 & 4.65 & 3.50 & 84.76 & 76.82 & 70.88 \\
\hline Giza 168 & 85.50 & 83.50 & 77.00 & 9.32 & 8.57 & 7.80 & 5.42 & 4.57 & 3.55 & 86.51 & 77.84 & 73.42 \\
\hline Gemmiza 11 & 89.83 & 84.83 & 79.00 & 9.91 & 8.88 & 8.68 & 5.23 & 3.94 & 3.42 & 85.97 & 75.42 & 71.67 \\
\hline Sids 12 & 86.50 & 82.83 & 78.17 & 5.46 & 4.52 & 3.98 & 5.82 & 5.05 & 3.96 & 87.35 & 78.62 & 71.36 \\
\hline Shandawel 1 & 85.33 & 79.17 & 74.67 & 12.38 & 9.46 & 8.74 & 5.20 & 4.46 & 3.56 & 87.56 & 78.28 & 74.71 \\
\hline Masr 1 & 80.83 & 78.83 & 74.17 & 9.78 & 7.67 & 7.26 & 5.42 & 4.31 & 3.39 & 84.73 & 75.04 & 66.29 \\
\hline Sahel 1 & 83.83 & 80.50 & 75.00 & 8.85 & 8.22 & 7.71 & 5.26 & 4.88 & 3.59 & 84.18 & 78.87 & 75.45 \\
\hline LSD0.05 & & 0.73 & & & 0.54 & & & 0.16 & & & 1.70 & \\
\hline
\end{tabular}

Egypt. J. Agron. 37, No. 2 (2015) 
TABLE 8. Mean performance of Chlorophyll concentration, WUE, grain yield / plant and Harvest index.

\begin{tabular}{|c|c|c|c|c|c|c|c|c|c|c|c|c|}
\hline \multirow[b]{2}{*}{ Genotypes } & \multicolumn{3}{|c|}{ Chlorophyll content } & \multicolumn{3}{|c|}{ WUE } & \multicolumn{3}{|c|}{ Grain yield / plant } & \multicolumn{3}{|c|}{ Harvest index } \\
\hline & \begin{tabular}{|c|} 
Well- \\
watered
\end{tabular} & \begin{tabular}{|c|} 
Mild \\
stress \\
\end{tabular} & \begin{tabular}{|c|}
$\begin{array}{c}\text { Sever } \\
\text { stress }\end{array}$ \\
\end{tabular} & \begin{tabular}{|c}
$\begin{array}{c}\text { Well- } \\
\text { watered }\end{array}$ \\
\end{tabular} & $\begin{array}{c}\text { Mild } \\
\text { stress }\end{array}$ & $\begin{array}{r}\text { Sever } \\
\text { stress }\end{array}$ & $\begin{array}{c}\text { Well- } \\
\text { watered }\end{array}$ & $\begin{array}{c}\text { Mild } \\
\text { stress } \\
\end{array}$ & $\begin{array}{l}\text { Sever } \\
\text { stress } \\
\end{array}$ & $\begin{array}{c}\text { Well- } \\
\text { watered }\end{array}$ & $\begin{array}{c}\text { Mild } \\
\text { stress } \\
\end{array}$ & $\begin{array}{l}\text { Sever } \\
\text { stress }\end{array}$ \\
\hline 1 & 465.33 & 328.51 & 249.42 & 1.11 & 0.79 & 0.66 & 24.71 & 17.51 & 14.76 & 37.63 & 34.95 & 34.84 \\
\hline 2 & 474.62 & 319.92 & 241.28 & 0.83 & 0.69 & 0.60 & 18.46 & 15.41 & 13.30 & 31.42 & 31.37 & 34.27 \\
\hline 3 & 322.94 & 193.15 & 147.28 & 0.73 & 0.57 & 0.53 & 16.34 & 12.78 & 11.85 & 28.47 & 32.02 & 32.82 \\
\hline 4 & 416.78 & 257.76 & 125.97 & 1.06 & 0.62 & 0.44 & 23.54 & 13.81 & 9.78 & 37.58 & 31.14 & 26.40 \\
\hline 5 & 409.93 & 298.21 & 211.40 & 0.90 & 0.70 & 0.62 & 20.04 & 15.48 & 13.84 & 40.82 & 37.76 & 37.61 \\
\hline 6 & 160.02 & 108.09 & 78.97 & 0.68 & 0.47 & 0.43 & 15.10 & 10.50 & 9.55 & 39.11 & 32.21 & 33.76 \\
\hline 7 & 491.27 & 276.84 & 162.43 & 0.79 & 0.54 & 0.47 & 17.56 & 11.94 & 10.44 & 36.16 & 33.08 & 32.65 \\
\hline 8 & 172.39 & 138.75 & 99.02 & 0.64 & 0.56 & 0.46 & 14.36 & 12.41 & 10.27 & 30.72 & 29.19 & 28.29 \\
\hline 9 & 402.78 & 212.25 & 100.31 & 1.01 & 0.61 & 0.39 & 22.48 & 13.57 & 8.60 & 35.94 & 33.15 & 27.46 \\
\hline 10 & 418.65 & 289.88 & 227.98 & 0.74 & 0.62 & 0.57 & 16.37 & 13.86 & 12.65 & 32.00 & 33.99 & 34.55 \\
\hline 11 & 616.43 & 418.98 & 320.75 & 1.16 & 0.72 & 0.64 & 25.91 & 16.13 & 14.29 & 39.55 & 37.29 & 38.00 \\
\hline 12 & 432.64 & 270.83 & 199.03 & 1.03 & 0.54 & 0.50 & 22.86 & 12.09 & 11.12 & 35.58 & 27.74 & 29.75 \\
\hline 13 & 217.23 & 126.64 & 109.10 & 0.80 & 0.51 & 0.48 & 17.90 & 11.31 & 10.68 & 35.37 & 30.72 & 33.03 \\
\hline 14 & 246.81 & 190.45 & 107.55 & 0.75 & 0.59 & 0.52 & 16.62 & 13.04 & 11.68 & 28.89 & 27.15 & 27.78 \\
\hline 15 & 353.45 & 232.09 & 176.37 & 0.81 & 0.53 & 0.48 & 17.98 & 11.82 & 10.62 & 29.74 & 29.02 & 31.02 \\
\hline 16 & 280.51 & 223.86 & 193.92 & 0.86 & 0.62 & 0.59 & 19.21 & 13.73 & 13.21 & 36.32 & 32.35 & 35.42 \\
\hline 17 & 255.67 & 161.32 & 119.85 & 0.83 & 0.53 & 0.47 & 18.57 & 11.83 & 10.53 & 33.63 & 28.90 & 30.91 \\
\hline 18 & 167.80 & 114.05 & 84.22 & 0.79 & 0.59 & 0.39 & 17.58 & 13.04 & 8.63 & 33.36 & 29.21 & 25.12 \\
\hline 19 & 453.17 & 301.58 & 232.47 & 0.78 & 0.69 & 0.62 & 17.28 & 15.46 & 13.90 & 29.44 & 27.71 & 29.41 \\
\hline 20 & 263.15 & 198.59 & 163.41 & 0.69 & 0.59 & 0.51 & 15.39 & 13.16 & 11.25 & 33.78 & 34.53 & 35.74 \\
\hline 21 & 215.46 & 136.71 & 80.65 & 0.70 & 0.46 & 0.35 & 15.58 & 10.25 & 7.82 & 33.18 & 28.49 & 25.91 \\
\hline 22 & 384.39 & 300.74 & 219.34 & 0.85 & 0.69 & 0.61 & 18.84 & 15.30 & 13.55 & 35.20 & 32.68 & 36.53 \\
\hline 23 & 378.04 & 264.89 & 230.46 & 0.79 & 0.64 & 0.60 & 17.68 & 14.34 & 13.45 & 34.79 & 32.28 & 34.63 \\
\hline 24 & 176.45 & 131.68 & 88.74 & 0.72 & 0.55 & 0.42 & 16.13 & 12.25 & 9.30 & 29.85 & 30.10 & 28.01 \\
\hline 25 & 195.46 & 136.41 & 101.65 & 0.73 & 0.60 & 0.46 & 16.35 & 13.28 & 10.31 & 32.83 & 32.14 & 30.37 \\
\hline 26 & 408.83 & 261.38 & 204.18 & 1.07 & 0.60 & 0.52 & 23.72 & 13.36 & 11.51 & 38.04 & 35.72 & 34.07 \\
\hline 27 & 473.11 & 234.91 & 153.05 & 1.00 & 0.54 & 0.42 & 22.21 & 12.06 & 9.29 & 39.38 & 28.26 & 28.67 \\
\hline 28 & 530.51 & 110.97 & 119.64 & 1.05 & 0.46 & 0.42 & 23.33 & 10.27 & 9.39 & 35.41 & 26.75 & 30.38 \\
\hline 29 & 301.40 & 193.68 & 156.09 & 0.77 & 0.58 & 0.56 & 17.04 & 12.88 & 12.29 & 28.41 & 27.56 & 30.36 \\
\hline 30 & 265.84 & 164.60 & 84.13 & 0.82 & 0.51 & 0.39 & 18.31 & 11.25 & 8.70 & 30.91 & 27.05 & 24.07 \\
\hline 31 & 413.13 & 256.86 & 186.90 & 0.82 & 0.56 & 0.42 & 18.31 & 12.55 & 9.37 & 33.45 & 32.21 & 28.72 \\
\hline 32 & 232.16 & 118.06 & 90.72 & 0.70 & 0.46 & 0.43 & 15.49 & 10.15 & 9.64 & 33.15 & 30.87 & 32.09 \\
\hline 33 & 174.13 & 125.15 & 95.52 & 0.70 & 0.57 & 0.42 & 15.52 & 12.73 & 9.41 & 30.38 & 30.97 & 29.64 \\
\hline 34 & 426.00 & 300.97 & 249.67 & 0.88 & 0.71 & 0.62 & 19.68 & 15.81 & 13.74 & 33.06 & 32.42 & 33.31 \\
\hline 35 & 194.72 & 129.94 & 84.03 & 0.87 & 0.53 & 0.48 & 19.35 & 11.78 & 10.64 & 35.55 & 31.42 & 31.63 \\
\hline 36 & 168.79 & 115.24 & 73.24 & 0.61 & 0.51 & 0.46 & 13.61 & 11.34 & 10.28 & 28.15 & 30.73 & 32.27 \\
\hline 37 & 462.19 & 311.97 & 235.52 & 0.96 & 0.61 & 0.56 & 21.39 & 13.69 & 12.41 & 38.43 & 35.09 & 36.00 \\
\hline 38 & 183.93 & 143.05 & 86.83 & 0.76 & 0.56 & 0.53 & 16.83 & 12.49 & 11.78 & 29.62 & 29.63 & 32.46 \\
\hline 39 & 576.92 & 121.45 & 76.36 & 0.96 & 0.46 & 0.40 & 21.46 & 10.18 & 8.88 & 34.81 & 29.51 & 29.34 \\
\hline 40 & 353.59 & 235.64 & 163.77 & 0.82 & 0.55 & 0.45 & 18.28 & 12.25 & 10.12 & 31.02 & 29.96 & 30.17 \\
\hline 41 & 496.08 & 356.63 & 270.03 & 1.28 & 0.71 & 0.66 & 28.50 & 15.84 & 14.60 & 40.85 & 40.14 & 40.55 \\
\hline 42 & 505.56 & 332.28 & 282.04 & 1.09 & 0.80 & 0.67 & 24.17 & 17.72 & 14.84 & 36.80 & 36.94 & 38.74 \\
\hline 43 & 476.86 & 291.84 & 281.74 & 0.88 & 0.73 & 0.62 & 19.69 & 16.28 & 13.71 & 33.39 & 31.78 & 33.74 \\
\hline 44 & 222.18 & 153.56 & 113.29 & 0.61 & 0.48 & 0.45 & 13.55 & 10.79 & 10.12 & 29.34 & 26.22 & 27.24 \\
\hline 45 & 173.99 & 114.67 & 85.58 & 0.63 & 0.48 & 0.45 & 14.04 & 10.66 & 10.00 & 30.73 & 28.65 & 30.89 \\
\hline 46 & 347.48 & 247.45 & 98.42 & 0.75 & 0.60 & 0.40 & 16.60 & 13.25 & 8.86 & 29.70 & 28.40 & 24.90 \\
\hline 47 & 163.64 & 99.33 & 69.63 & 0.52 & 0.44 & 0.33 & 11.54 & 9.77 & 7.43 & 29.18 & 30.81 & 29.62 \\
\hline 48 & 352.72 & 227.07 & 140.36 & 0.69 & 0.54 & 0.45 & 15.40 & 12.02 & 9.97 & 33.52 & 32.37 & 34.99 \\
\hline 49 & 222.92 & 158.07 & 110.05 & 0.69 & 0.53 & 0.49 & 15.34 & 11.89 & 10.90 & 30.39 & 29.31 & 39.31 \\
\hline 50 & 277.18 & 209.27 & 144.80 & 0.86 & 0.62 & 0.54 & 19.25 & 13.92 & 11.92 & 33.75 & 32.05 & 32.23 \\
\hline Mean & 335.50 & 212.92 & 154.54 & 0.83 & 0.58 & 0.50 & 18.51 & 12.98 & 11.10 & 33.58 & 31.28 & 31.79 \\
\hline Giza 168 & 408.54 & 279.79 & 194.30 & 0.80 & 0.63 & 0.57 & 17.78 & 14.13 & 12.67 & 30.15 & 27.73 & 30.25 \\
\hline Gemmiza 11 & 414.57 & 281.48 & 189.57 & 0.78 & 0.56 & 0.46 & 17.28 & 12.38 & 10.13 & 36.03 & 31.20 & 29.67 \\
\hline Sids 12 & 459.55 & 295.37 & 194.62 & 0.89 & 0.60 & 0.55 & 19.92 & 13.35 & 12.14 & 37.26 & 33.97 & 36.08 \\
\hline Shandawel 1 & 424.60 & 304.75 & 218.47 & 0.84 & 0.59 & 0.55 & 18.64 & 13.09 & 12.30 & 30.97 & 30.30 & 35.13 \\
\hline Masr 1 & 424.60 & 199.22 & 114.00 & 0.74 & 0.54 & 0.42 & 16.40 & 11.94 & 9.25 & 32.39 & 29.36 & 26.42 \\
\hline Sahel 1 & 375.12 & 248.89 & 212.38 & 0.76 & 0.62 & 0.57 & 16.88 & 13.78 & 12.69 & 34.02 & 32.11 & 35.15 \\
\hline LSD0.05 & & 13.86 & & & 0.04 & & & 0.90 & & & 1.96 & \\
\hline
\end{tabular}

Egypt. J. Agron. 37, No. 2 (2015) 
As an average for all genotypes WUE reduced by $29 \%$ and $39 \%$ under mild and severe drought conditions, respectively (Table 8). Results showed that line No. 19 recorded the lowest reduction in WUE (12\% and 21\%, respectively) under mild and severe drought conditions. On the other hand Line No. 28 was the most sensitive one (56\% reduction) under mild drought conditions followed by line No. 9 under severe drought conditions (61\% reduction). Six Lines (No. 1, $5,11,41,42$ and 43) surpassed the check (Sahel 1) and local commercial varieties in WUE over all conditions. Meanwhile, seven lines, i.e., No. 2, 10, 16, $19,22,23$ and 34 surpassed the check (Sahel 1) and local commercial varieties in WUE under mild and severe stresses conditions (Table 8).

Manifestly, the evaluated wheat genotypes in this study showed significant differences in their grain yield/ plant (Table 1). As an average for overall tested genotypes, grain yield/plant $(\mathrm{g})$ was reduced from 18.51 in well-watered conditions to 12.98 in mild conditions and further down to 11.1 in severe conditions. Clearly, severe drought stress was strong for grain yield / plant to be reduced by $40 \%$ average reduction (Table 8 ). The highest grain yield/ plant (g) was obtained from lines No. 1, 5, 11, 41 and 42 which were significantly outyielded the check (Sahel 1) and local commercial varieties under all conditions (Table 8).

Harvest index ranged from $28.15 \%$ to $40.85 \%$ with an average of $33.56 \%$ under well-watered condition (Table 8). While, under mild stress it ranged from $26.22 \%$ to $40.14 \%$ with a reduction on general mean by $6.97 \%$ as compared with well-watered condition. Moreover, under severe condition the mean harvest index for all genotypes was $31.83 \%$ recording the reduction by $5.17 \%$ as compared with well-watered condition. Results revealed that lines, i.e., No. 1, 4, $5,6,11,26,27,37$ and 41 surpassed the check (Sahel 1) and local commercial varieties in harvest index under all conditions (Table 8).

The drought susceptibility index (DSI) for the tested lines shown in Table 9. DSI over mild stress condition ranged from 0.35 for line No. 19 to 1.88 for line No. 28 and twenty nine lines gave low values of drought susceptibility index (DSI < 1). Meanwhile, under severe stress condition the values of DSI ranged from 0.49 for line No. 19 to 1.55 for line No. 9 and twenty seven lines have DSI $<1$. Finally, results indicated that eight lines, i.e., No. 2, 5, 19, 22, 23, 34, 42 and 43 produced relatively high grain yield compared with the check (Sahel 1) and local commercial varieties under drought stress environments due to high yield potential, rather than having DSI $<1$ (Table 9). 
TABLE 9. Mean performance of grain yield / plant and drought susceptibility index.

\begin{tabular}{|c|c|c|c|c|c|c|}
\hline \multirow[b]{2}{*}{ Genotypes } & \multicolumn{2}{|c|}{ Grain yield/ plant } & \multirow[b]{2}{*}{ DSImild } & \multicolumn{2}{|c|}{ Grain yield/ plant } & \multirow[b]{2}{*}{ DSI Isever } \\
\hline & $\begin{array}{c}\text { Well- } \\
\text { watered }\end{array}$ & $\begin{array}{c}\text { Mild } \\
\text { stress }\end{array}$ & & $\begin{array}{c}\text { Well- } \\
\text { watered }\end{array}$ & $\begin{array}{c}\text { Sever } \\
\text { stress }\end{array}$ & \\
\hline 1 & 24.71 & 17.51 & 0.98 & 24.71 & 14.76 & 1.01 \\
\hline 2 & 18.46 & 15.41 & 0.56 & 18.46 & 13.30 & 0.70 \\
\hline 3 & 16.34 & 12.78 & 0.73 & 16.34 & 11.85 & 0.69 \\
\hline 4 & 23.54 & 13.81 & 1.39 & 23.54 & 9.78 & 1.47 \\
\hline 5 & 20.04 & 15.48 & 0.76 & 20.04 & 13.84 & 0.78 \\
\hline 6 & 15.10 & 10.50 & 1.02 & 15.10 & 9.55 & 0.92 \\
\hline 7 & 17.56 & 11.94 & 1.07 & 17.56 & 10.44 & 1.02 \\
\hline 8 & 14.36 & 12.41 & 0.46 & 14.36 & 10.27 & 0.72 \\
\hline 9 & 22.48 & 13.57 & 1.33 & 22.48 & 8.60 & 1.55 \\
\hline 10 & 16.37 & 13.86 & 0.51 & 16.37 & 12.65 & 0.57 \\
\hline 11 & 25.91 & 16.13 & 1.27 & 25.91 & 14.29 & 1.13 \\
\hline 12 & 22.86 & 12.09 & 1.58 & 22.86 & 11.12 & 1.29 \\
\hline 13 & 17.90 & 11.31 & 1.24 & 17.90 & 10.68 & 1.01 \\
\hline 14 & 16.62 & 13.04 & 0.72 & 16.62 & 11.68 & 0.75 \\
\hline 15 & 17.98 & 11.82 & 1.15 & 17.98 & 10.62 & 1.03 \\
\hline 16 & 19.21 & 13.73 & 0.96 & 19.21 & 13.21 & 0.78 \\
\hline 17 & 18.57 & 11.83 & 1.22 & 18.57 & 10.53 & 1.09 \\
\hline 18 & 17.58 & 13.04 & 0.87 & 17.58 & 8.63 & 1.28 \\
\hline 19 & 17.28 & 15.46 & 0.35 & 17.28 & 13.90 & 0.49 \\
\hline 20 & 15.39 & 13.16 & 0.49 & 15.39 & 11.25 & 0.68 \\
\hline 21 & 15.58 & 10.25 & 1.15 & 15.58 & 7.82 & 1.25 \\
\hline 22 & 18.84 & 15.30 & 0.63 & 18.84 & 13.55 & 0.71 \\
\hline 23 & 17.68 & 14.34 & 0.63 & 17.68 & 13.45 & 0.60 \\
\hline 24 & 16.13 & 12.25 & 0.81 & 16.13 & 9.30 & 1.06 \\
\hline 25 & 16.35 & 13.28 & 0.63 & 16.35 & 10.31 & 0.93 \\
\hline 26 & 23.72 & 13.36 & 1.47 & 23.72 & 11.51 & 1.29 \\
\hline 27 & 22.21 & 12.06 & 1.53 & 22.21 & 9.29 & 1.46 \\
\hline 28 & 23.33 & 10.27 & 1.88 & 23.33 & 9.39 & 1.50 \\
\hline 29 & 17.04 & 12.88 & 0.82 & 17.04 & 12.29 & 0.70 \\
\hline 30 & 18.31 & 11.25 & 1.29 & 18.31 & 8.70 & 1.32 \\
\hline 31 & 18.31 & 12.55 & 1.06 & 18.31 & 9.37 & 1.23 \\
\hline 32 & 15.49 & 10.15 & 1.16 & 15.49 & 9.64 & 0.95 \\
\hline 33 & 15.52 & 12.73 & 0.60 & 15.52 & 9.41 & 0.99 \\
\hline 34 & 19.68 & 15.81 & 0.66 & 19.68 & 13.74 & 0.76 \\
\hline 35 & 19.35 & 11.78 & 1.31 & 19.35 & 10.64 & 1.13 \\
\hline 36 & 13.61 & 11.34 & 0.56 & 13.61 & 10.28 & 0.61 \\
\hline 37 & 21.39 & 13.69 & 1.21 & 21.39 & 12.41 & 1.06 \\
\hline 38 & 16.83 & 12.49 & 0.87 & 16.83 & 11.78 & 0.75 \\
\hline 39 & 21.46 & 10.18 & 1.76 & 21.46 & 8.88 & 1.47 \\
\hline 40 & 18.28 & 12.25 & 1.11 & 18.28 & 10.12 & 1.12 \\
\hline 41 & 28.50 & 15.84 & 1.49 & 28.50 & 14.60 & 1.23 \\
\hline 42 & 24.17 & 17.72 & 0.90 & 24.17 & 14.84 & 0.97 \\
\hline 43 & 19.69 & 16.28 & 0.58 & 19.69 & 13.71 & 0.76 \\
\hline 44 & 13.55 & 10.79 & 0.68 & 13.55 & 10.12 & 0.64 \\
\hline 45 & 14.04 & 10.66 & 0.81 & 14.04 & 10.00 & 0.72 \\
\hline 46 & 16.60 & 13.25 & 0.68 & 16.60 & 8.86 & 1.17 \\
\hline 47 & 11.54 & 9.77 & 0.51 & 11.54 & 7.43 & 0.89 \\
\hline 48 & 15.40 & 12.02 & 0.74 & 15.40 & 9.97 & 0.89 \\
\hline 49 & 15.34 & 11.89 & 0.75 & 15.34 & 10.90 & 0.73 \\
\hline 50 & 19.25 & 13.92 & 0.93 & 19.25 & 11.92 & 0.96 \\
\hline
\end{tabular}

\section{Molecular analysis of wheat lines under drought}

A comparison between the highest and lowest 10 bulked-lines in some agronomic traits performance evaluated under drought conditions was achieved. Ten SRAP primers used in the analysis showed the difference between the highest and lowest lines by generating unique and specific bands for each bulk (Table 10, Fig. 1). The total number of unique and specific bands ranged from 5 to 14 bands per trait, while the average percentage of polymorphism $(\% \mathrm{P})$ ranged from 4.07 to $10.37 \%$ for yield and water use efficiency (WUE), respectively (Table 10).

Egypt. J. Agron. 37, No. 2 (2015) 
SRAP MARKERS ASSOCIATED WITH WATER ...

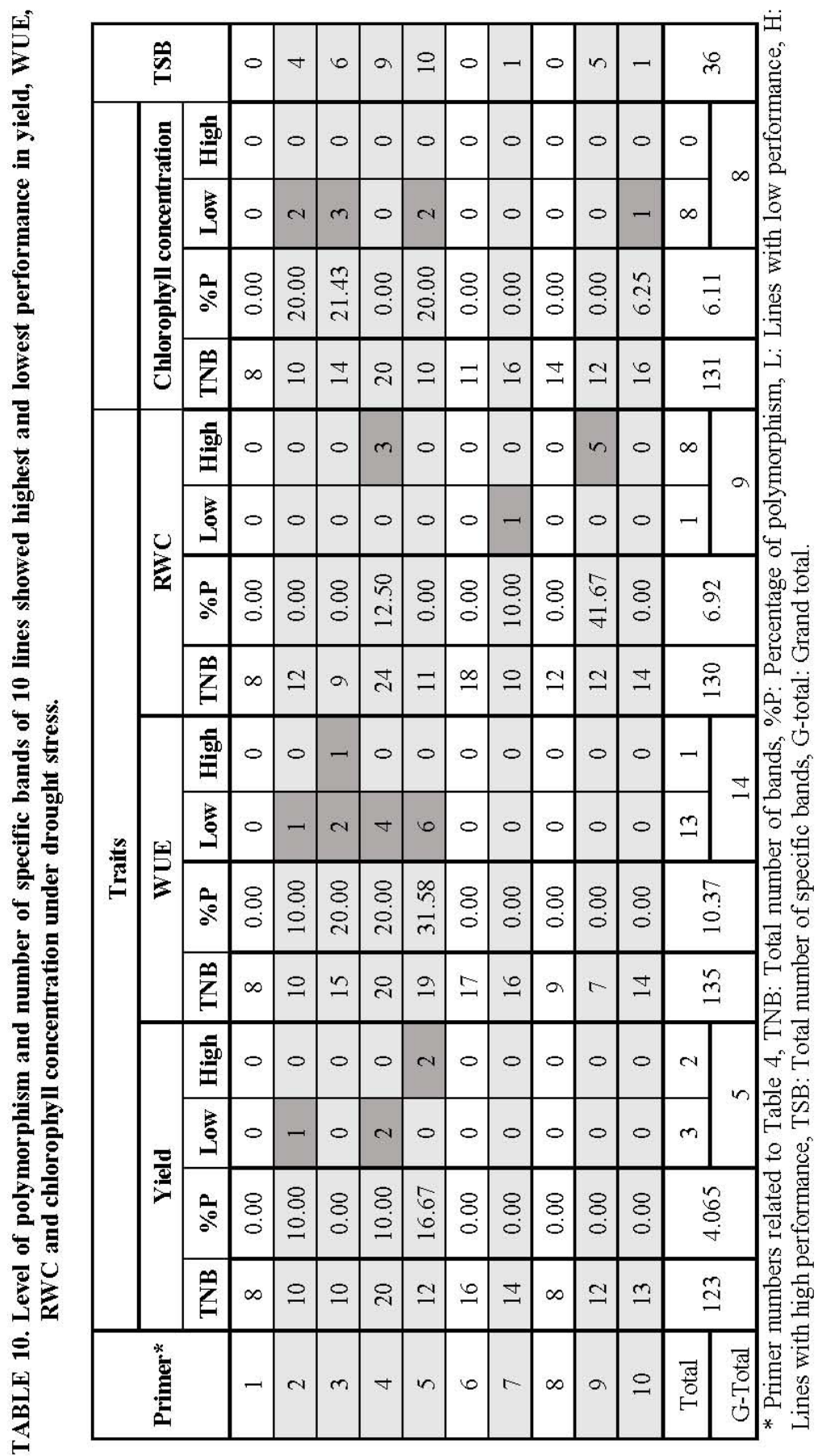

Egypt. J. Agron. 37, No. 2 (2015) 

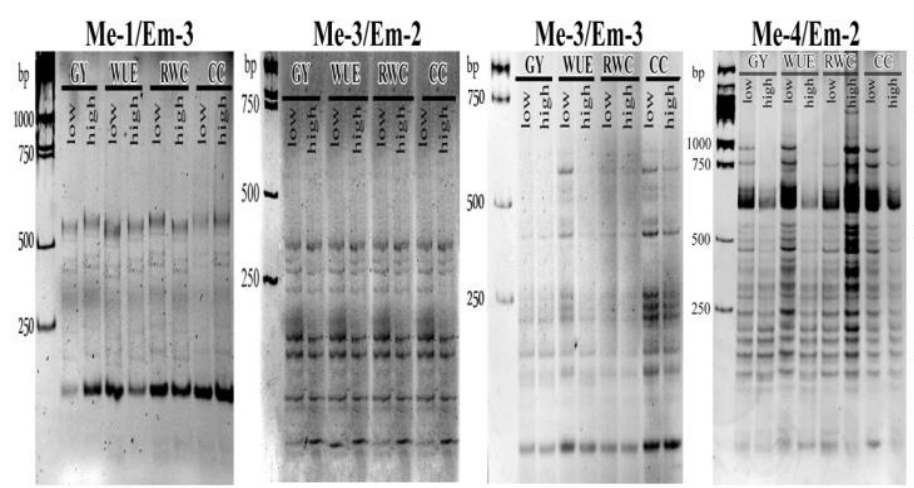

$\mathrm{Me}-4 / \mathrm{Em}-3$
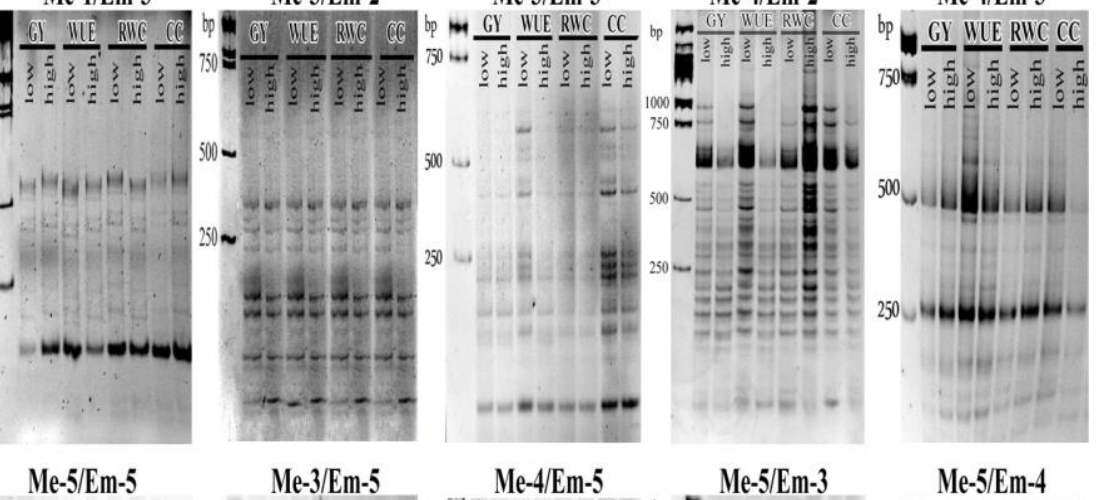

Me-3/Em-5

Me-4/Em-5

Me-5/Em-3

$\mathrm{Me}-5 / \mathrm{Em}-4$

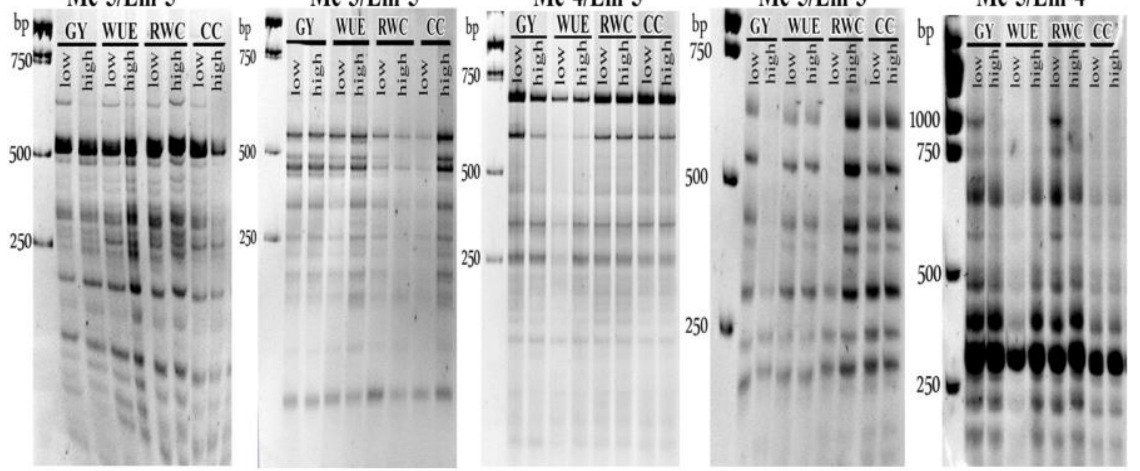

Fig. 1. SRAP profile of 10 primer combinations showing the difference between two bulks of 10-high and low performance wheat lines in some traits evaluated under drought stress; GY: Grain yield per plant, WUE: Water use efficiency, RWC: Relative water content, CC: Chlorophyll concentration, high: Bulk of the highest 10 lines and low: Bulk of the lowest 10 lines.

The total number of bands generated for high- and low- bulked lines for yield, water use efficiency (WUE), relative water content (RWC) and chlorophyll concentration was 123, 135, 130 and 131 bands, respectively. A total of 36 bands were generated uniquely and specific for the evaluated traits (Tables 10 and 11). For instance, 5 bands were generated for high- and low-yield bulked lines (2 and 3, respectively) with $4.07 \%$ polymorphism. The total number of specific bands in the case of WUE was 14, out of them 13 were specific for bulked-lines with low WUE and one band for bulked-lines with high WUE with $10.37 \%$ polymorphism. In addition, nine specific bands were generated for WRC lines, out of them 8 were specific for high WRC bulked-lines, while one band was specific for low WRC with $6.92 \%$ polymorphism. Furthermore, eight bands were specific for lines with high chlorophyll concentration with an average of $6.11 \%$ polymorphism, while no specific bands were generated for low chlorophyll concentration lines (Table 10). 
TABLE 11. Size in base pair of specific bands for tow bulks of 10 lines showed highest and lowest performance in Yield, WUE, RWC and chlorophyll concentration under drought stress.

\begin{tabular}{|c|c|c|c|c|c|c|c|c|c|}
\hline \multirow{3}{*}{ 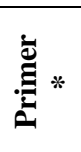 } & \multicolumn{8}{|c|}{ Traits } & \multirow{3}{*}{ NSB } \\
\hline & \multicolumn{2}{|c|}{ Yield } & \multicolumn{2}{|c|}{ WUE } & \multicolumn{2}{|c|}{ RWC } & \multicolumn{2}{|c|}{$\begin{array}{c}\text { Chlorophyll } \\
\text { concentration }\end{array}$} & \\
\hline & Low & High & Low & High & Low & High & Low & High & \\
\hline 2 & 295 & - & 295 & - & - & - & $\begin{array}{l}295 \\
230\end{array}$ & - & 4 \\
\hline 3 & - & - & $\begin{array}{l}560 \\
595\end{array}$ & 140 & - & - & $\begin{array}{l}380 \\
530 \\
650 \\
\end{array}$ & - & 6 \\
\hline 4 & $\begin{array}{l}750 \\
920\end{array}$ & - & $\begin{array}{c}400 \\
750 \\
920 \\
1100\end{array}$ & - & - & $\begin{array}{c}580 \\
920 \\
1050\end{array}$ & - & - & 9 \\
\hline 5 & - & $\begin{array}{l}305 \\
340\end{array}$ & $\begin{array}{l}775 \\
735 \\
705 \\
655 \\
640 \\
615\end{array}$ & - & - & - & $\begin{array}{l}305 \\
380\end{array}$ & - & 10 \\
\hline 7 & - & - & - & - & 185 & - & - & - & 1 \\
\hline 9 & - & - & - & - & - & $\begin{array}{l}365 \\
400 \\
450 \\
530 \\
655\end{array}$ & - & - & 5 \\
\hline 10 & - & - & - & - & - & - & 600 & - & 1 \\
\hline NSB & 3 & 2 & 13 & 1 & 1 & 8 & 8 & 0 & 36 \\
\hline
\end{tabular}

* Primer numbers related to table 4, NSB: number of specific bands

\section{Discussion}

The selection for some physiological traits such as water use efficiency (WUE), relative water content (RWC), chlorophyll concentration in drought conditions studies are important for improving drought tolerance in wheat (Larbi, 2004 and Shirazi et al., 2014). In the present study, drought caused significant reduction in all evaluated agronomic traits. In this regard, drought stress at grain filling period adversely affected 100 -kernel weight, as it is more critical phase and results in substantial yield losses (Muhammad et al., 2014). Moreover, in our study, water stress caused a reduction on RWC which reflected the effect of drought stress and exposed the difference among the evaluated wheat genotypes. In this regard, Shamsi (2010) reported that plants which can reserve more water content on its tissues have a good performance in drought conditions. In addition, the tested genotypes showed significant differences in chlorophyll concentration which considered as an indicator for the yield stability under drought conditions (Sairam, 1994 and Shamsi, 2010). 
To get the maximum output from wheat genotypes, we should evaluate the genetically improved varieties for growth performance and water use efficiency (WUE) under drought conditions (Shirazi et al., 2014). In the present study, WUE was used to evaluate the tested lines and showed higher reduction under both mild and severe drought conditions. Additionally, it was reported that genotype which can use water more efficiently produce maximum biomass (Shirazi et al., 2014).

Grain yield is frequently used in crops such as wheat as the main criteria for drought resistance (Li et al., 2011). The reduction showed in the present study in grain yield due to water deficit is supported by that such trait is affected by a complex of different morphological and physiological characters which are in turn influenced by the soil moisture (Sodabeh et al., 2013). Moreover, under water limited conditions, genotypes which show the highest harvest index and yield stability were reported as drought tolerant (Rathore, 2005).

In general, our results are in agreement with those of Li et al. (2011) who studied the effect of drought stress on some agronomic characteristics and grain yield in spring wheat. They found that drought caused obvious reductions in several traits including plant height, grain volume weight, kernel weight and diameter and grain yield. Furthermore, El-Rawy \& Youssef (2014) found that drought conditions generated by the treatment of polyethylene glycol caused high reduction in shoot and root lengths in some wheat lines evaluated at seedling stage.

On the other hand, the association of molecular markers with phenotypic evaluationis one of important factors to understand and investigate the genetic role of tolerance by prediction the genomic regions that affect the plant's response (Roy et al., 2011). Therefore, identification of molecular bands associated with some traits evaluated under stress is the most important step in selecting genotypes having tolerance to such trait at the early stages of growth. In addition, molecular markers can improve the efficiency of breeding by allowing manipulation of the genome through marker-assisted selection (Prerna et al., 2013).

In the present study, SRAP marker was able to differentiate among different bulked-DNA of high and low performance in all agronomic traits evaluated under drought stress. The dissimilarities within each group was reduced by gathering their DNA samples, therefore the difference between the high and low bulks was mainly due to the trait of interest. Moreover, SRAP showed its effectiveness by generating several specific bands for the tolerant and susceptible bulked-lines. The generated bands could serve preliminarily as selectable markers for drought tolerance in wheat; however purification, sequencing and analysis of these bands might be necessary in the proximate research work.

The previous studies on wheat diversity and genotyping indicated that SRAP was an efficient technique for wheat diversity evaluation. In this regard, Zaefizadeh \& Goleiv (2009) investigated the genetic diversity and relationships

Egypt. J. Agron. 37, No. 2 (2015) 
among durum wheat landraces from the region of North West Iran and Azerbaijan by SRAP marker and phenotypic differences. They found that 12 combinations of SRAP markers were distinguishable among these landraces, they suggested that SRAP technology is useful for genetic diversity and relationship analyses, marker assisted selection and genetic map construction in durum wheat. On the other hand, Elshafei et al. (2013) used SRAP markers to identify new markers linked to chlorophyll concentration, flag leaf senescence and cell membrane stability in wheat under water stress conditions; they reported that SRAP generated successfully several QTLs linked to these traits. In addition, Moustafa et al. (2014) reported that, TRAP and SRAP markers, combined with bulked segregant analysis, could be used to identify molecular markers linked to six agronomic traits; (days to heading, plant height, spike number $/ \mathrm{m}^{2}$, kernel number/spike, 1000-kernel weight and grain yield), as indicators for drought tolerance genes in wheat. Recently, El-Rawy \& Youssef (2014) reported the efficiency of SRAP in discriminating wheat genotypes under drought conditions at seedling stage using polyethylene glycol. They found that SRAP generated up to $85.71 \%$ polymorphism among the tested lines as well as SRAP showed its effectiveness by gathering all lines which have a high DSI in one sub-cluster and generated several unique and specific bands for high-DSIlines and other for low-DSI-lines, suggesting that these bands could be used for further work as SRAP markers associated with drought tolerance in wheat.

Our results in generating unique bands specific for certain traits evaluated under drought stress were in accordance with previous studies. In this regards, the primer combination used in our study (4- ACC/TGC) which generated 10 specific bands specific for low-yield ( 2 bands), low-WUE (4 bands), high-RWC (3 bands) and low-AO (one band), has been reported to generate QTL specific for flag leaf senescence (Elshafei et al., 2013) and to generate 3 bands specific for lines with high drought susceptibility index (DSI) and one band for low-DSIlines (El-Rawy \& Youssef, 2014). Moreover, the primer combination 9AAG/GAC which generated 5 bands specific for high-RWC lines in our study has been reported as well to generate QTL specific for flag leaf senescence (Elshafei et al., 2013).

\section{Conclusion}

Drought caused significant reduction in all evaluated agronomic traits. Comparing the two bulks of the highest and lowest performance lines in some agronomic traits evaluated under drought stress molecularly was sufficient to expose the difference between the tolerant and susceptible lines. SRAP showed its effectiveness in discriminating the tested genotypes by generating several unique and specific bands for high and/or low-performance in some agronomic traits evaluated under water stress. These bands could be identified as markers associated with drought tolerance in wheat. Additionally, evaluation of WUE in this study showed its efficiency in differentiating among the tested lines and was in agreement with SRAP analysis which showed the maximum number of specific markers when the high- and low-WUE bulks were compared unlike the other traits.

Egypt. J. Agron. 37, No. 2 (2015) 
Acknowledgments: The authors wish to thank the Regional Councils for Agricultural Research and Extension, Ministry of Agriculture and Land Reclamation, Egypt for the financial support.

\section{Reference}

Allen, R.G., Pereira, L.S., Raes, D. and Smith, M. (1998) "Crop Evapotranspiration. Guidelines for Computing Crop Water Requirements", Irrigation and Drainage Paper 56, Food and Agric. Organization of the United Nations, Rome, Italy, 300 pp

Dash, J., Curran, P. J., Tallis， M. J., Llewellyn, G. M., Taylor, G. and Snoeij, P. (2007) The relationship between the MERIS terrestrial chlorophyll index and chlorophyll content. Second ENVISAT Symposium, Europian Space Agency. Noordwijk, ESA, SP-636 (CD-Rom).

Dellaporta, S.L., Wood, J. and Hicks, J.B. (1983) A plant DNA mini-preparation: version II. Plant Mol. Biol. Rep. 1, 19-21.

El-Rawy, M.A. and Youssef, M. (2014) Evaluation of drought and heat tolerance in wheat based on seedling traits and molecular analysis. J. Crop Sci. Biotech. 17(3),183-189. doi:10.1007/s12892-014-0053-x.

Elshafei, A.A., Saleh, M., Al-Doss, A.A., Moustafa, K.A., Al-Qurainy, F.H. and Barakat, M.N. (2013) Identification of new SRAP markers linked to leaf chlorophyll content, flag leaf senescence and cell membrane stability traits in wheat under waterstressed condition. Aust. J. Crop Sci. 7, 887-893.

FAOSTAT (2010) Food and Agriculture Organization of the United Nations. http://faostat.fao.org.

FAOSTAT (2012) Food and Agriculture Organization of the United Nations. http://faostat.fao.org.

Hittalmani, S., Huang, N., Courtois, B., Venuprasad, R., Shashidhar, H. E., Zhuang J. Y., Zheng, K. L., Liu, G. F., Wang, G. C., Sidhu, J. S., Srivantaneeyakul, S., Singh, V. P., Bagali, P. G., Prasanna, H. C., McLaren, G., and Khush, G. S. (2003) Identification of QTL for growth- and grain yield-related traits in rice across nine location of Asia. Theor. Appl. Genet. 107, 679-690.

Klute, A. (ed.) (1986) "Methods of Soil Analysis. Part 1 - Physical and Mineralogical Methods". $2^{\text {nd }}$ ed. SSSA Book Series No. 5. SSSA and ASA, Madison, WI.

Larbi, A. (2004) Relative water content (RWC) and leaf senescence as screening tools for drought tolerance in wheat. In : "Mediterranean Rainfed Agriculture: Strategies for Sustainability" . Cantero-Martínez, C. and Gabiña, D. (Ed.). pp. 193-196, Zaragoza CIHEAM.

Li, G. and Quiros, C.F. (2001) Sequence-related amplified polymorphism (SRAP), a new marker system based on a simple PCR reaction: its application to mapping and gene tagging in Brassica. Theor. Appl. Genet. 103, 455-461.

Egypt. J. Agron. 37, No. 2 (2015) 
Li, P., Chen, J. and Wu, P. (2011) Agronomic characteristics and grain yield of 30 spring wheat genotypes under drought stress and nonstress conditions. Agronomy Journal, 103 (6), 1619-1628.

Ludlow, M. M. and Muchow, R. C. (1990) A critical evaluation of the traits for improving crop yield in water limited environments. Adv. Agron. 43,107-153.

Moustafa, K.A., Saleh, M., Al-Doss, A.A., Elshafei, A.A., Salem, A.K., Al-Qurainy, F.H. and Barakat, M.N. (2014) Identification of TRAP and SRAP markers linked with yield components under drought stress in wheat (Triticum aestivum L.). Plant Omics Journal, 7(4), 253-259.

Muhammad, F., Mubshar, H. and Kadambot, H.M.S. (2014) Drought stress in wheat during flowering and grain-filling periods. Critical Reviews in Plant Sciences, 33(4), 331-349.

Nazari, L. and Pakniyat, H. (2010) Assessment of drought tolerance in barley genotypes. Journal of Applied Sciences, 10(2), 151-156.

Page, A. L. (ed.) (1982) "Methods of Soil Analysis, Part 2-Chemical and Microbiological Properties", Second edition.

Prerna, Kumar, A. and Sengar, R.S. (2013) Evaluation of heat and drought tolerance of wheat cultivars through physiological, biochemical and molecular approaches. Research Journal of Agricultural Sciences, 4(2), 139-145.

Rathore, P.S. (2005) "Techniques and Management of Field Crop Production". Agribios, India, p. 525.

Roy, S.J., Tucker, E.J. and Tester, M. (2011) Genetic analysis of abiotic stress tolerance in crops. Current Opinion in Plant Biol. 14, 232-239.

Sairam, R.K.(1994) Effect of moisture stress on physiological activities of two contrasting wheat genotypes. Indian J. Exp. Biol. 32, 594-597

Shamsi, K. (2010) The effects of drought stress on yield, relative water content, proline, soluble carbohydrates and chlorophyll of bread wheat cultivars. Journal of Animal \& Plant Sciences, 8(3), 1051- 1060.

Shirazi, M.U., Khan, M.A., Bhatti, N., Unar, A., Bozdar, H.B., Mujtaba, S.M., and Lashari, M.I. (2014) Growth and water use efficiency in wheat genotypes grown under water stress condition. Journal of Agricultural Research and Development, 4(2), 023-028.

Sodabeh, R., Davoud, H., Marefat, G. and Nasser, M. (2013) Evaluation of tolerant to drought stress after anthesis in promising winter and facultative wheat genotypes under field condition. Intl. J. Agron. Plant. Prod. 4 (2), 171-177.

Stulnig, T.M. and Amberger, A. (1994) Exposing contaminating phenol in nucleic acid preparations. BioTech. 16, 403-404. 
Trethowan, R. M. and Pfeiffer, W. H. (2000) Challenges and future strategies in breeding wheat for adaptation to drought stressed environments: A CIMMYT wheat program perspective. In: "Molecular Approaches for the Genetic Improvement of Cereals for Stable Production in Water-limited Environments". Ribaut. J.M. Poland D (Ed.), pp 45-48. A strategic planning workshop held at CIMMYT El Batan, Mexico, 21-25 June 1999. CIMMYT, Mexico DF.

Vites, F.G.Jr. (1965) Increasing water efficiency by soil management. Amer. Soci. Agron. 26, 259-274

Youssef, M. (2012) Khirshyat 1.0: A simple micro-program for some molecular biology protocols. Genes, Genomes and Genomics, 6,102-105.

Zaefizadeh, M., Goliev, R. (2009) Diversity and relationships among durum wheat landraces (subconvars) by SRAP and phenotypic marker polymorphism. Res. J. Biol. Sci. 4, 960-966.

(Received 7/7/2015;

accepted 28/9/2015) 


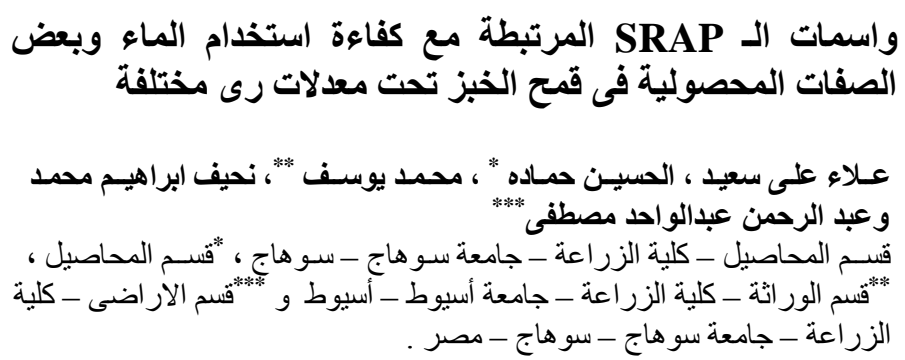

تم تقييم 50 سلالة من قمح الخبز للتحمل للجفاف و مقارنتهم بستة أصناف محلية

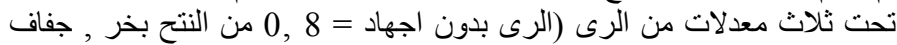

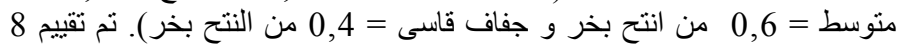

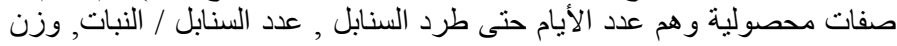

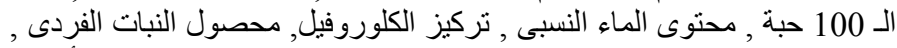

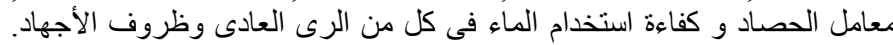

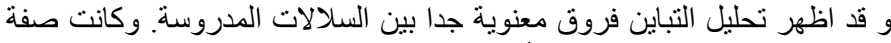

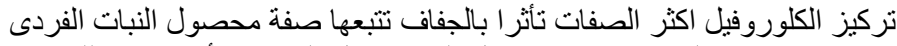

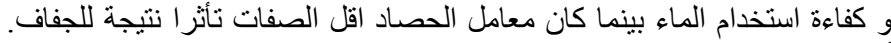

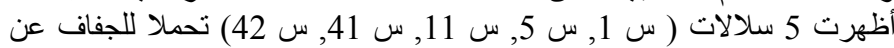

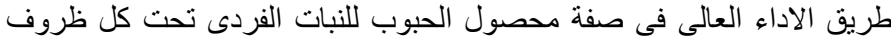

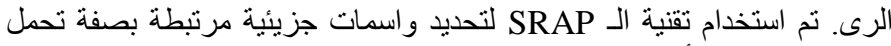

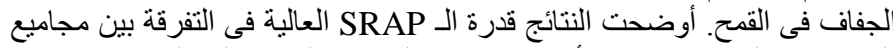

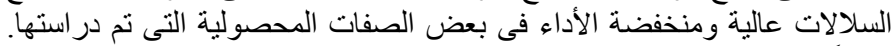

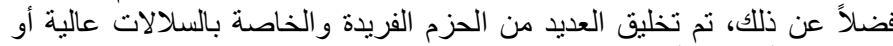

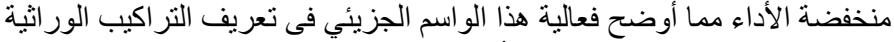

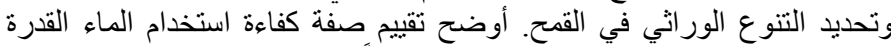

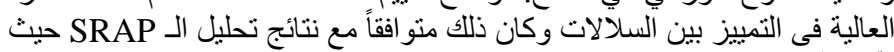

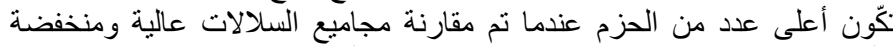

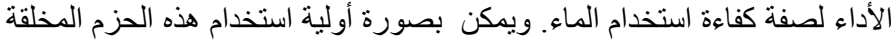

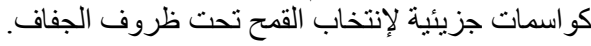

